\title{
Aging and Crossovers in Phase-Separating Fluid Mixtures
}

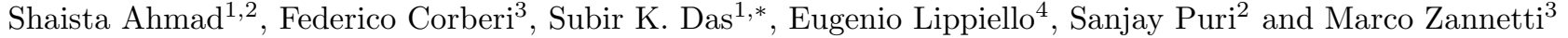 \\ ${ }^{1}$ Theoretical Sciences Unit, Jawaharlal Nehru Centre for Advanced Scientific Research, Jakkur P.O., Bangalore 560064, India \\ ${ }^{2}$ School of Physical Sciences, Jawaharlal Nehru University, New Delhi 110067, India \\ ${ }^{3}$ Dipartimento di Matematica e Informatica and CNISM, Unità di Salerno, \\ Università di Salerno, via Ponte don Melillo, 84084 Fisciano (SA), Italy \\ ${ }^{4}$ Dipartimento di Scienze Ambientali, Seconda Università di Napoli, Via Vivaldi, Caserta, Italy
}

(Dated: November 4, 2018)

\begin{abstract}
We use state-of-the-art molecular dynamics simulations to study hydrodynamic effects on aging during kinetics of phase separation in a fluid mixture. The domain growth law shows a crossover from a diffusive regime to a viscous hydrodynamic regime. There is a corresponding crossover in the autocorrelation function from a power-law behavior to an exponential decay. While the former is consistent with theories for diffusive domain growth, the latter results as a consequence of faster advective transport in fluids for which an analytical justification has been provided.
\end{abstract}

PACS numbers: 29.25.Bx. 41.75.-i, 41.75.Lx

\section{INTRODUCTION}

There has been intense recent interest in understanding the coarsening dynamics of phase-separating mixtures [1-13]. For fluid systems, attention has focused on single-time quantities, e.g., the correlation function and structure factor, or the growth law of the domain scale $\ell(t)[2,10,14,15]$. An equally important and deeply related aspect of coarsening systems is the nature of the aging properties [16] which are encoded in two-time quantities. This issue has been intensively studied in the context of disordered systems and structural glasses, in phase-separating systems without hydrodynamic modes, such as solid mixtures, but not in segregating fluids. In part, this is due to the scarcity of reliable numerical results for these systems. In this letter, we present the first molecular dynamics (MD) results for aging in coarsening binary fluid. Before discussing our model and results, it is useful to give a brief overview of the main concepts.

Following a quench inside the miscibility gap, a homogeneous binary mixture $(\mathrm{A}+\mathrm{B})$ separates into $\mathrm{A}$-rich and B-rich phases. The evolution of the system from the randomly-mixed phase to the segregated state is a complex process. Domains rich in A- and B-particles form and grow nonlinearly with time [14, 15, 17, 18]. This coarsening is a self-similar process which is reflected in the scaling behavior of various physical quantities, as the two-point equal-time correlation function $G(r, t)=$ $\left\langle\phi\left(\vec{r}_{1}, t\right) \phi\left(\vec{r}_{2}, t\right)\right\rangle-\left\langle\phi\left(\vec{r}_{1}, t\right)\right\rangle\left\langle\phi\left(\vec{r}_{2}, t\right)\right\rangle$ (where the order parameter $\phi$ is the difference between the A and B concentrations and $\left.r=\left|\vec{r}_{1}-\vec{r}_{2}\right|\right)$. This quantity can be expressed in the scaling form $G(r, t)=\tilde{G}(r / \ell), \tilde{G}(z)$ being a time-independent master function. Typically, $\ell(t)$ grows in a power-law manner as $\ell(t) \sim t^{\alpha}$, where the growth exponent $\alpha$ depends upon the transport mechanism. For diffusive domain growth [19] $\alpha=1 / 3$, which is referred to as the Lifshitz-Slyozov law. This behavior characterizes segregating solid mixtures. However in fluids, hydrodynamic transport mechanisms, important at large length scales, lead to much faster growth. In spatial dimension $d=3$, for a percolating domain morphology, advective transport results in three growth regimes [20]:

$$
\alpha= \begin{cases}1 / 3, & \ell(t) \ll \ell_{d v}=(D \eta)^{1 / 2}, \\ 1, & \ell_{d v} \ll \ell(t) \ll \ell_{i n}=\eta^{2} /(\rho \gamma), \\ 2 / 3, & \ell(t) \gg \ell_{i n} .\end{cases}
$$

In Eq. (11), $D$ is the diffusion constant, $\eta$ the viscosity, $\rho$ is the density, $\gamma$ the surface tension, $\ell_{d v}$ is the crossover length from diffusive to viscous regime, and $\ell_{i n}$ the one from viscous to inertial hydrodynamic regime [15].

For diffusive phase-separation, also the orderparameter auto-correlation function $C\left(t, t_{w}\right)=$ $\left\langle\phi(\vec{r}, t) \phi\left(\vec{r}, t_{w}\right)\right\rangle-\langle\phi(\vec{r}, t)\rangle\left\langle\phi\left(\vec{r}, t_{w}\right)\right\rangle$ (where $t$ and the waiting time $t_{w}<t$ are two generic instants) takes a scaling form $C\left(t, t_{w}\right)=\tilde{C}\left(\ell / \ell_{w}\right)$ where hereafter $\ell=\ell(t)$ and $\ell_{w}=\ell\left(t_{w}\right)$. The scaling function behaves as $\tilde{C}(x)=x^{-\lambda}$ for large $x=\ell / \ell_{w}, \lambda$ being the Fisher-Huse (FH) exponent 21] whose value in $d=3$, according to the results in Ref. [22], is in the range $[1.5,1.6]$. Despite this good understanding of the aging properties in diffusive systems, the behavior in segregating fluids remains largely unknown. In this letter we start filling the gap by studying the properties of the autocorrelation function in a range of times encompassing the first two regimes of Eq. (11) in a model of binary fluid. In doing that we uncover an interesting structure with a crossover which corresponds to the switch from the early diffusive to 
the intermediate hydrodynamic regime in Eq. (1) for the growth law. Specifically, we find the two parameter scaling form

$$
C\left(t, t_{w}, \ell_{d v}\right)=\tilde{C}(x, y),
$$

where $x=\ell / \ell_{w}, y=\ell_{d v} / \ell_{w}$, and $\tilde{C}(x, y)$ obeys the limiting behaviors:

$$
\tilde{C}(x, y)= \begin{cases}x^{-\lambda}(\text { with } \lambda \simeq 1.5), & y \gg 1 \\ e^{-(x-1) / \tau}, & y \ll 1 .\end{cases}
$$

Thus, in the diffusive regime $\left(\ell_{w} \ll \ell_{d v}\right.$ or $\left.y \gg 1\right)$, we obtain a power-law decay of the scaling function as for spin systems. To the best of our knowledge, this has not been observed in previous studies of fluid phase separation. Furthermore, and more importantly, in the hydrodynamic regime $\left(\ell_{w} \gg \ell_{d v}\right.$ or $\left.y \ll 1\right)$, we find a crossover to a novel exponential. The latter finding is the central result of this letter. Here $y \gg 1$ and $y \ll 1$ should be read as nearly pure diffusive and pure hydrodynamic regimes, respectively.

The rest of the paper is organized as follows. We describe the model and methodology in Section II. Results are presented in Section III. Finally, Section IV concludes the paper with a brief summary.

\section{MODEL AND METHOD}

We consider a periodic box of linear dimension $L$ containing $\mathrm{A}$ and $\mathrm{B}$ particles of equal mass $m$. Particles $i$ and $j$, at distance $r$ apart, interact via the potential

$$
\left\{\begin{array}{l}
u(r)=U(r)-U\left(r_{c}\right)-\left(r-r_{c}\right)\left(\frac{d U}{d r}\right)_{r=r_{c}} ; r<r_{c}, \\
u(r)=0 ; r>r_{c} .
\end{array}\right.
$$

Here, the Lennard-Jones pair potential $U(r)$ has the form $U(r)=4 \varepsilon_{\alpha \beta}\left[(\sigma / r)^{12}-(\sigma / r)^{6}\right]$, where $\sigma$ is the interaction diameter, and $\varepsilon_{\alpha \beta}$ is the interaction strength between particles of species $\alpha$ and $\beta[\alpha, \beta \in(A, B)]$. The potential in Eq. (4) is cut-off at $r=r_{c}$, to ensure faster computation [23, 24]. For convenience, we set $r_{c}=2.5 \sigma$. The third term on the right-hand-side of Eq. (4), after a shifting of the potential by its value at $r=r_{c}$ (see the second term), was introduced to make both the potential and force continuous at $r=r_{c}$. The overall density $\rho$ is set to unity so that the fluid is incompressible. For the choice $\varepsilon_{A A}=\varepsilon_{B B}=2 \varepsilon_{A B}=\varepsilon$, we have an Ising-like fully symmetric model. The phase behavior and equilibrium properties of this system, that exhibits a liquid-liquid phase transition at a critical temperature $k_{B} T_{c} \simeq 1.423 \varepsilon$, are well studied [25]. For convenience, we set $\varepsilon, k_{B}, m$ and $\sigma$ to unity below.
Our choice of a high density ensures that the gas-liquid transition is well separated from the liquid-liquid one.

We employ this model to study the kinetics of phase separation via MD simulations [23, 24] by quenching homogeneous critical (50\% A and $50 \%$ B particles) configurations, prepared at a very high temperature $(T=10)$, to temperatures below $T_{c}$. We have used the Verlet velocity algorithm with integration time step $\Delta t=0.01 \tau$, where the time unit $t_{0}=\left(m \sigma^{2} / \varepsilon\right)^{1 / 2}=1$. The temperature was controlled via application of a Nosé-Hoover thermostat 24], that is well-known to preserve hydrodynamics.

While unambiguous confirmation of expected hydrodynamic effects, via MD simulation, in binary fluid phase separation was done only recently, by using this model [2, 26], signature of such fast hydrodynamic mechanism was observed in a number of earlier MD simulations [27-29].

For analysis of our results, we mapped the continuum fluid configurations onto a simple cubic lattice of size $L^{3}$ [2]. There each site $i$ is occupied by a particle and we assign a spin value $S_{i}=+1$ if it is an A-particle and $S_{i}=-1$ elsewhere. This mapping ensures that the pattern dynamics can be studied in a manner analogous to the spin-1/2 Ising model. The two-point equaltime correlation function was calculated as $G(r, t)=$ $\left\langle S_{i}(t) S_{j}(t)\right\rangle-\left\langle S_{i}(t)\right\rangle\left\langle S_{j}(t)\right\rangle$, with $r=|i-j|$ and the angular brackets denote an averaging over independent runs. For a conserved order-parameter system, $G(r, t)$ exhibits damped oscillations around zero. The average domain size $\ell(t)$ is obtained from the first zero crossing of $G(r, t)$. The auto-correlation function was calculated as $C\left(t, t_{w}\right)=\left\langle S_{i}(t) S_{i}\left(t_{w}\right)\right\rangle-\left\langle S_{i}(t)\right\rangle\left\langle S_{i}\left(t_{w}\right)\right\rangle$. All results presented subsequently for $T=1.1=0.77 T_{c}$ correspond to $L=64$, whereas those for $T=1.25=0.88 T_{c}$ were obtained for $L=48$. For statistical quantities, we average over 10 independent runs.

\section{RESULTS}

Figure 1 shows snapshots from the evolution of the binary fluid. The snapshot at $t=0$ corresponds to the homogeneously mixed phase immediately after the quench to the temperature $T=1.1$. The other snapshots show the growth of bicontinuous A-rich and Brich domains - this continues until the system reaches equilibrium.

To obtain a quantitative understanding of the evolution, in Fig. 2 we plot $\ell(t)$ for two different temperatures [2]. The inset shows the collapse of data for $C(r, t)$ from different times upon scaling the abscissa by $\ell(t)$. The behavior of $\ell(t)$ is consistent with the expected diffusive 

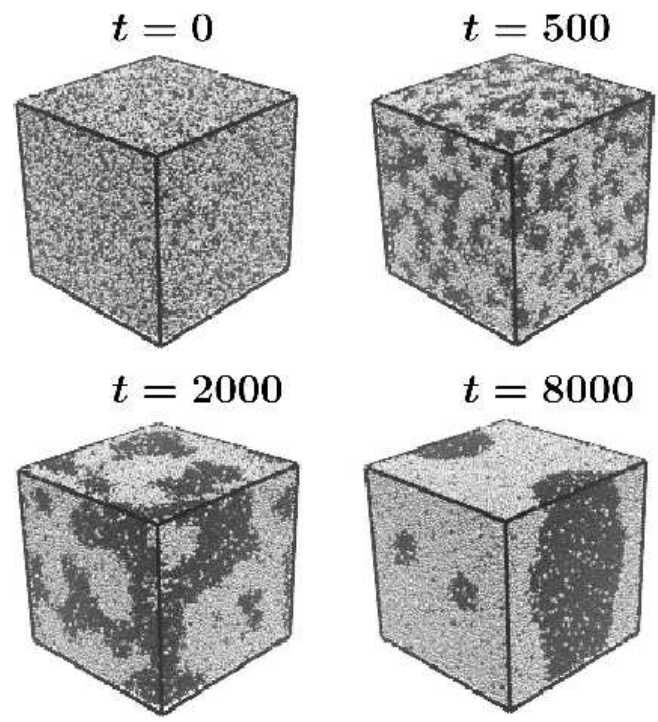

FIG. 1: Evolution snapshots obtained after a quench to a temperature $T=1.1$. The A-particles are marked black, B-particles are gray.

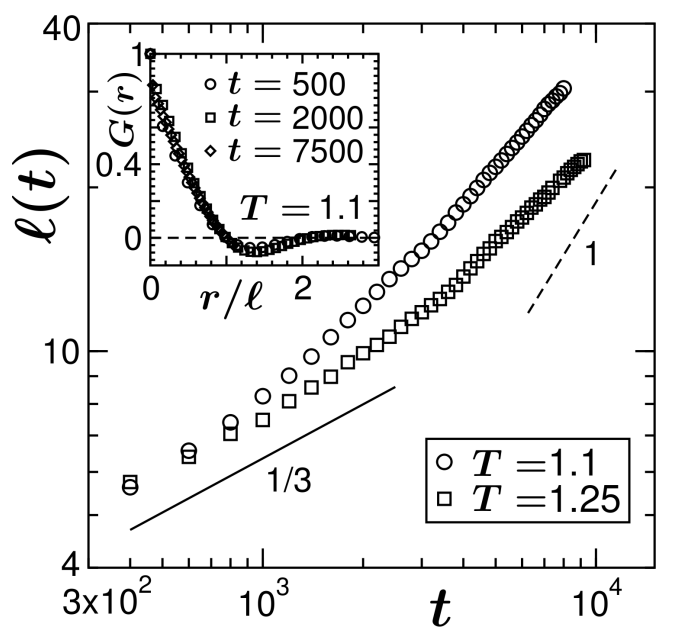

FIG. 2: Growth $\ell(t)$ as a function of time for temperatures $T=1.1$ and $T=1.25$, on a log-log scale. The solid and dashed lines correspond to diffusive $(\alpha=1 / 3)$ and viscous hydrodynamic $(\alpha=1)$ growth laws, respectively. Inset: Scaling plot of $G(r, t)$ from three different times for $T=1.1$.

behavior (with $\alpha=1 / 3$ ) at early times, with a crossover to a faster growth later on. As discussed in [26], the second regime is consistent with linear hydrodynamic growth at later times. Indeed the slightly off-parallel nature (on a log-log plot) of the late-time data from the linear behavior is due to non-zero off-sets at the crossovers that can be conveniently subtracted off to obtain a genuine linear behavior [26]. We observe that the crossover between the two growth regimes is seen to occur earlier for lower temperatures, since diffusion is enhanced by thermal fluctuations.

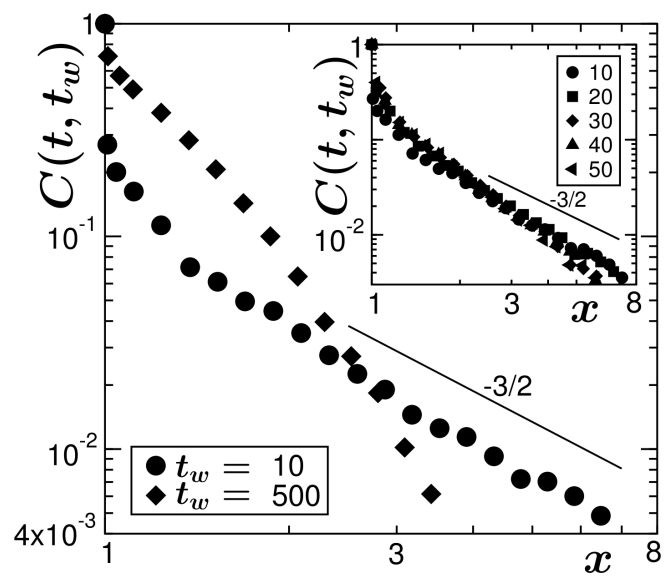

FIG. 3: Plot of $C\left(t, t_{w}\right)$ vs. $x=\ell(t) / \ell\left(t_{w}\right)$ for $T=1.1$ and different values of $t_{w}$ (Main: $t_{w}=10,500$, Inset: $t_{w}=$ $10,20,30,40,50)$, on a log-log scale. The solid lines denote the power-law behavior $x^{-3 / 2}$.

As stated earlier, our primary objective is to study aging phenomena, essentially the behavior of $C\left(t, t_{w}\right)$. In Fig. 3. we plot $C\left(t, t_{w}\right)$ as a function of $x=\ell(t) / \ell\left(t_{w}\right)$ for different $t_{w}$. It is observed that, for the smaller values of $t_{w}$, presented in the inset, there is a reasonable collapse of data, not perfect though, and the mastercurve obeys the form $\tilde{C}(x) \sim x^{-\lambda}$, with $\lambda \simeq 1$.5. This is the expected behavior for phase-separation in the diffusive stage. We must add here the reason for the imperfect collapse of data which will also explain the logic behind the choice of a narrow range of $t_{w}$ to demonstrate this power-law scaling behavior. The height at the beginning of the power-law regime changes with $t_{w}$, the increase of which takes the domain order-parameter closer to the equilibrium value. Further, with the increase of $t_{w}$, faster decay due to hydrodynamics (see below) shows up earlier in $x$. Due to this latter fact the data sets as a whole give the impression of an exponent steeper than the expected value. However, from the results for $t_{w}=10$ in the main frame, consistencey with the FH exponent should be appreciated.

On the other hand, for larger values of $t_{w}$, e.g. $t_{w}=500$ in the main frame, a crossover from the FH behavior is observed, where, in the post crossover regime the decay of $C\left(t, t_{w}\right)$ is much faster than a power law. In order to check for a possible exponential decay at large $t_{w}$, a semi-log plot is presented in Fig. 4(a). In this figure, the values of $t_{w}$ were judicially chosen such that for time beyond this, diffusive mechanism is practically negligible compared to the influence of hydrody- 


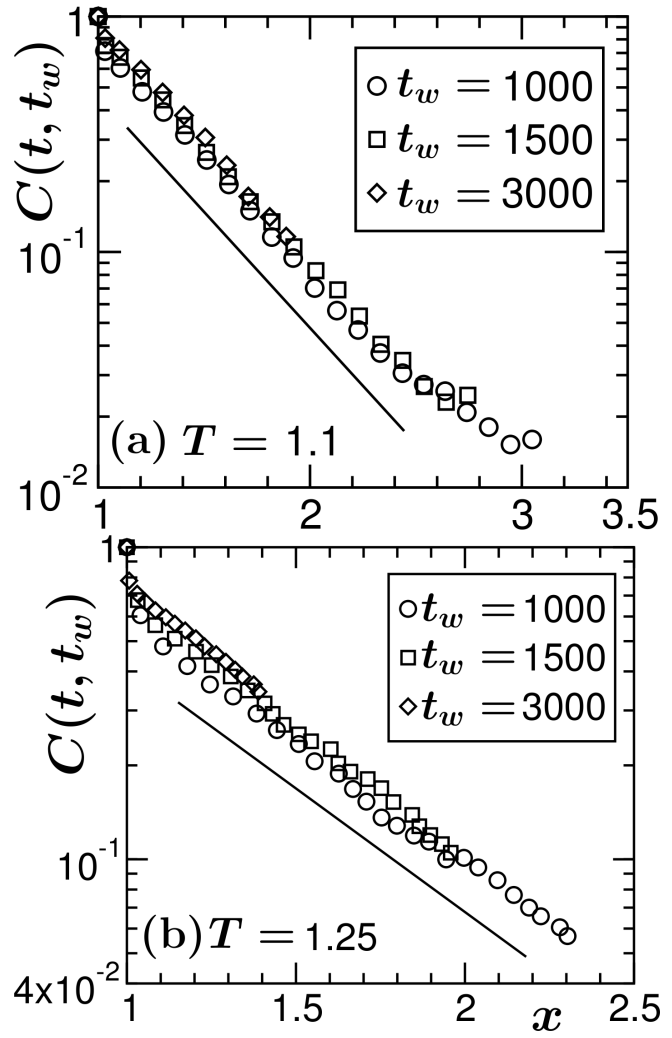

FIG. 4: (a) Same as Fig 3 but on a semi-log plot and for $t_{w}=1000,1500,3000$. (b) As in (a) but at $T=1.25$.

namics. The smallest value of $t_{w}$ in this figure, viz., $t_{w}=1000$, was chosen being motivated by a finite-size scaling study [26] that showed a pure viscous hydrodynamic behavior for time beyond this. Interestingly, in this post-crossover regime a scaling form is recovered, as proven by the excellent data collapse. Moreover, the behavior of the scaling function $\tilde{C}(x)$ is very nicely consistent with an exponential decay. A similar behavior, with scaling and exponential decay of $\tilde{C}(x)$ is also observed for $T=1.25$ (Fig. 4(b)), showing that the whole pattern is rather general. Here we note that the rate of decay, defined by $\tau$, in this hydrodynamic regime should depend upon the interfacial tension and the advection field (influenced by transport properties like shear viscosity). This will be justified towards the end of this paper. As is clear from parts (a) and (b) of Fig 4 this decay rate is a temperature dependent quantity.

The deviation from a linear look, observed for $T=1.1$ at very large values of $x$, occur in a range of time where finite-size effects have been observed [26]. Indeed, as it can be recognized in Fig. 1, for such times the size of domains is comparable to the system size, the liquid is close to equilibrium and thus the configurations change very slowly. For this reason, we are unable to access the inertial hydrodynamic regime $[\alpha=2 / 3$ in Eq. (1)] which would require significantly larger system sizes and is beyond our present computational resources.

We attribute this exponential decay of the autocorrelation function to a fast advective field in the hydrodynamic regime which is explained below. The continuum dynamical equation [Cahn-Hilliard], used to study kinetics of diffusive phase separation in binary alloys, can be modified to [15]

$$
\frac{\partial \phi}{\partial t}+\vec{v} \cdot \vec{\nabla} \phi=D \nabla^{2} \mu
$$

to investigate hydrodynamic effects in fluid phase separation. Here $\vec{v}$ is the time and space dependent velocity field that also has dependence upon shear viscosity as well as other transport properties and $\mu$ is the chemical potential. In the regime where hydrodynamics is the dominant mechanism, the right hand side of Eq. (5) (that takes care of diffusion) can be neglected to write

$$
\frac{\partial \phi}{\partial t}+\vec{v} \cdot \vec{\nabla} \phi=0
$$

Assuming $\vec{v}$ to be a constant and representing $\phi$ in the $k$-space, one obtains the solution

$$
\phi(\vec{k}, t)=\phi\left(\vec{k}, t_{w}\right) e^{i \vec{v} \cdot \vec{k}\left(t-t_{w}\right)} .
$$

Hence for the $k$-space autocorrelation $\mathcal{C}(\vec{k}, t)$ [= $\left.\left\langle\phi(\vec{k}, t) \phi\left(-\vec{k}, t_{w}\right)\right\rangle\right]$, one has

$$
\mathcal{C}\left(\vec{k}, t, t_{w}\right)=\mathcal{G}\left(\vec{k}, t_{w}\right) e^{i \vec{v} \cdot \vec{k}\left(t-t_{w}\right)},
$$

where $\mathcal{G}\left(\vec{k}, t_{w}\right)=\mathcal{C}\left(\vec{k}, t_{w}, t_{w}\right)$ is the equal-time structure factor for time $t_{w}$. The autocorrelation function then is

$$
\begin{aligned}
C\left(t, t_{w}\right)=\int d \vec{k} \mathcal{C}\left(\vec{k}, t, t_{w}\right) & =\int d \vec{k} \mathcal{G}\left(\vec{k}, t_{w}\right) e^{i \vec{v} \cdot \vec{k}\left(t-t_{w}\right)} \\
& =G\left(\vec{r}=\vec{v}\left(t-t_{w}\right), t_{w}\right),(9)
\end{aligned}
$$

where $G\left(\vec{r}, t_{w}\right)$ is the (real space) correlation function at time $t_{w}$. Recalling the scaling property $G(\vec{r}, t)=$ $\tilde{G}\left(\frac{r}{\ell(t)}\right)$, one arrives at

$$
C\left(t, t_{w}\right)=\tilde{G}\left(\frac{v\left(t-t_{w}\right)}{\ell\left(t_{w}\right)}\right) .
$$

For small values of $x$, Porod's law [15] states that $\tilde{G}(x) \simeq 1-a x$, where $a$ is a constant. This is consistent with an exponential decay for small $x$. The question then is what happens for large values of $x$. Here note that an analytical form of $\tilde{G}(x)$, for conserved orderparameter dynamics, still remains a challenging task. However, our analysis of the numerical data [see the inset of Fig 2 is suggestive of an exponential decay of the two-point equal time correlation function for large enough values of $x$. 


\section{CONCLUSION}

We have undertaken the first study of aging dynamics during phase separation in a binary Lennard-Jones fluid. The average domain size grows in a power-law fashion, with an exponent $\alpha=1 / 3$ (diffusive) at early times and $\alpha=1$ (viscous hydrodynamic) at later times. This crossover has a remarkable consequence for the scaling form of the autocorrelation function $C\left(t, t_{w}\right)$. In the diffusive regime, $C\left(t, t_{w}\right)$ shows a power-law decay with the Fisher-Huse exponent. However, in the hydrodynamic regime, we observe an exponential decay. This is due to the fact that advective hydrodynamic flows wash out the memory very rapidly by displacing the domains. We believe that the results presented here will arise fresh experimental and theoretical interest in this problem.

\section{Acknowledgments:}

SKD and SA acknowledge grant number SR/S2/RJN-13/2009 of the Department of Science and Technology, India. SKD also acknowledges hospitality at the University of Salerno, Italy. SA is grateful to the University Grants Commission for partial support and JNCASR for supporting her extended visits.

*das@jncasr.ac.in
[1] D. Reith, K. Bucior, L. Yelash, P. Virnau and K. Binder, J. Phys: Cond. Mat. 24, 115102 (2012).

[2] S. Ahmad, S.K. Das and S. Puri, Phys. Rev. E 82, 040107 (2010); ibid 85, 031140 (2012).

[3] S. Majumder and S.K. Das, Phys. Rev. E 81, 050102 (2010); ibid 84, 021110 (2011).

[4] R. Khanna, N.K. Agnihotri, M. Vashistha, A. Sharma, P.K. Jaiswal and S. Puri, Phys. Rev. E 82, 011601 (2010).

[5] E. Lippiello, A. Mukherjee, S. Puri and M. Zannetti, Europhys. Lett. 90, 46006 (2010). F. Corberi, E. Lippiello, A. Mukherjee, S. Puri and M. Zannetti, J. Stat. Mech., P03016 (2011); Phys. Rev. E 85, 021141 (2012).

[6] M.J.A. Hore and M. Laradji, J. Chem. Phys. 132, 024908 (2010).

[7] K. Bucior, L. Yelash and K. Binder, Phys. Rev. E 77, 051602 (2008).

[8] A. Sicilia, Y. Sarrazin, J.J. Arenzon, A.J. Bray and L.F. Cugliandolo, Phys. Rev. E 80, 031121 (2009).

[9] N. Blondiaux, S. Morgenthaler, R. Pugin, N. D. Spencer and M. Liley, Appl. Surf. Sci. 254, 6820 (2008); J. Liu, $\mathrm{X}$. Wu, W. N. Lennard and D. Landheer, Phys. Rev. B 80, 041403 (2009).

[10] S.J. Mitchell and D.P. Landau, Phys. Rev. Lett. 97, 025701 (2006).

[11] S.K. Das, S. Puri, J. Horbach and K. Binder, Phys. Rev. E 72, 061603 (2005); ibid 73, 031604 (2006); Phys. Rev. Lett. 96, 016107 (2006).

[12] C. Castellano, F. Corberi and M. Zannetti, Phys. Rev. E 56, 4973 (1997). F. Corberi and C. Castellano, ibid 58, 4658 (1998). C. Castellano and F. Corberi, ibid 57, 672 (1998); ibid 61, 3252 (2000); Phys. Rev. B 63, 060102 (2001).

[13] F. Corberi, E. Lippiello and M. Zannetti, Phys. Rev. E 65, 046136 (2002); ibid 78, 011109 (2008). F. Corberi, A. Coniglio and M. Zannetti, ibid 51, 5469 (1995).

[14] S. Puri and V. Wadhawan (Eds.), Kinetics of Phase Transitions (CRC Press, Boca Raton, FL, 2009).

[15] A.J. Bray, Adv. Phys. 51, 481 (2002).
[16] J.P. Bouchaud, L.F. Cugliandolo, J. Kurchan and M. Mezard in Spin Glasses and Random fields, Directions in Condensed Matter Physics Vol. 12, p. 161, A.P. Young (Ed.) (World Scientific, Singapore, 1998). F. Corberi, L.F. Cugliandolo, H. Yoshino, in Dynamical heterogeneities in glasses, colloids, and granular media, Eds.: L. Berthier, G. Biroli, J-P Bouchaud, L. Cipelletti and W. van Saarloos (Oxford University Press, 2011). M. Zannetti, in [14], p. 153.

[17] K. Binder, in Phase Transformation of Materials, Vol. 5, p. 405, R.W. Cahn, P. Haasen and E.J. Kramer (Eds.) (VCH, Weinheim, 1991).

[18] R.A.L. Jones, Soft Condensed Matter (Oxford University Press, Oxford, 2008). A. Onuki, Phase Transition Dynamics (Cambridge University Press, Cambridge, 2002).

[19] I.M. Lifshitz and V.V. Slyozov, J. Phys. Chem. Solids 19, 35 (1961).

[20] E.D. Siggia, Phys. Rev. A 20, 595 (1979). H. Furukawa, Phys. Rev. A 31, 1103 (1985); ibid 36, 2288 (1987).

[21] D.A. Huse, Phys. Rev. B 40, 304 (1989). D.S. Fisher and D.A. Huse, Phys. Rev. B 38, 373 (1989).

[22] F. Liu, and G.F. Mazenko, Phys. Rev. B 44, 9185 (1991); C. Yeung, M. Rao and R.C. Desai, Phys. Rev. E 53, 3073 (1996); G.F. Mazenko, Phys. Rev. E 58, 1543 (1998).

[23] M.P. Allen and D.J. Tildesley, Computer Simulations of Liquids, (Clarendon, Oxford, 1987).

[24] D. Frankel and B. Smit, Understanding Molecular Simulations: From Algorithms to Application (Academic Press, San Diego, California, 2002).

[25] S.K. Das, M.E. Fisher, J.V. Sengers, J. Horbach and K. Binder, Phys. Rev. Lett. 97, 025702 (2006). S.K. Das, J. Horbach, K. Binder, M.E. Fisher and J. V. Sengers, J. Chem. Phys. 125, 024506 (2006). S. Roy and S.K. Das, Europhys. Lett. 94, 36001 (2011).

[26] S.K. Das, S. Roy, S. Majumdar, and S. Ahmad, Europhys. Lett. 97, 66006 (2012).

[27] M. Laradji, S. Toxvaerd and O.G. Mouritsen, Phys. 
Rev. Lett. 77, 2253 (1996).

[28] H. Kabrede and R. Hentschke, Physica A 361, 485 (2006).
[29] A.K. Thakre, W.K. den Otter and W.J. Briels, Phys. Rev. E 77, 011503 (2008). 